\title{
Orígenes del sistema de cooperación internacional tras la II Guerra Mundial: Las campañas de inmunización contra la tuberculosis. De Europa a la India de Nehru, 1944-1960
}

Origins of the international cooperation system after World War II. Immunization campaigns against tuberculosis, from Europe to Nehru's India, 1944-1960

Mariano Monge Juárez

Universidad de Murcia

\section{RESUMEN}

En el seno de una Europa destruida y asediada por las epidemias, surge la idea de afrontar la amenaza de la tuberculosis con una gran campaña de BCG en Alemania, Austria, Polonia, Yugoslavia, Hungría o India. Este artículo trata de sintetizar el devenir de hechos, condiciones y causas implícitas en el desarrollo de estas grandes campañas contra la tuberculosis, que, impulsadas desde la Dinamarca, Suecia y Noruega, pronto reciben el apoyo de la OMS y UNICEF, y llegan a inmunizar a más veinte millones de personas en India. La campaña contra la tuberculosis sienta las bases del futuro de erradicación de la viruela, también de la cooperación internacional durante las últimas décadas del siglo XX. Los hechos son paradigmáticos de una nueva biopolítica en Europa.

PALABRAS CLAVE: cooperación, UNICEF, OMS, tuberculosis, biopolítica

\section{ABSTRACT}

Within a Europe destroyed and besieged by epidemics, the idea of tackling the threat of tuberculosis arises with a large BCG campaign in Germany, Austria, 
Poland, Yugoslavia, Hungary or India. This article tries to synthesize the facts, conditions and causes implicit in the development of these great campaigns against tuberculosis, which, promoted by Denmark, Sweden and Norway, soon receive the support of $\mathrm{WHO}$ and UNICEF, and become immunized to over twenty million people in India. The campaign against tuberculosis lays the foundations for the future of smallpox eradication, as well as international cooperation during the last decades of the 20th century. The facts are paradigmatic of a new biopolitics in Europe.

KEY WORDS: cooperation, UNICEF, WHO, tuberculosis, biopolitics

\section{OBJETIVO}

Este artículo se ocupa se sintetizar la historia de las campañas de vacunación masiva contra la tuberculosis que la Cruz Roja danesa, junto a otras organizaciones noruegas y suecas, impulsaron con la financiación y coordinación de la OMS y UNICEF. La "International Tuberculosis Compaign" (ITC) -también conocida como "Joint enterprice"- se desarrolló entre 1948 y 1960 en las regiones de Europa más afectadas por las consecuencias de la II Guerra Mundial, tales como Polonia, Yugoslavia, Hungría, Austria o Checoslovaquia, y posteriormente en India, Egipto, China o México entre otros países.

Los objetivos que nos planteamos son tres: presentar un breve estado de la cuestión sobre los trabajos que hasta el momento se ha ocupado de estas campañas masivas internacionales de vacunación contra la tuberculosis con BCG (Bacilo Calmette-Guérin) en Europa e India; ubicar su significado histórico en el contexto de los años de la posguerra mundial y despegue económico del viejo continente; y analizar los resultados y significados que produce la "Joint enterprice" en cuanto a la historia y la sociología de las organizaciones no gubernamentales y otras instituciones equivalentes.

\section{MATERIAL}

El material que ha servido para esta investigación ha sido por una parte las fuentes primarias, es decir, documentos que aportan información directa de las campañas de la ITC, emitidos entre 1949 y 1960, de entre los que cabe destacar los informes de la OMS firmados por Johannes Holm o Halfdan Mahler sobre el desarrollo de la "Joint entreprice" en Europa e India respectivamente. En cuanto 
a las fuentes secundarias, es necesario llamar la atención sobre los estudios de Niels Brimnes o Christian Bonah, que aportan un interesante y pormenorizado relato de los hechos.

\section{MÉTODOS}

El artículo ha seguido un método cualitativo, que se ha desarrollado a lo largo de cuatro fases: la lectura de las fuentes secundarias, el análisis de las fuentes primarias teniendo muy en cuenta las contribuciones de la bibliografía existente, la contextualización de los hechos, y la interpretación historiográfica de los documentos desde una óptica hermenéutica, en la que planteamos tanto las cuestiones condicionales y contextuales como etiológicas de la "International Tuberculosis Compaign" para llegar a una serie de conclusiones generales que pretenden aportan un nuevo análisis de las campañas.

\section{EL CONTEXTO: DE LA EUROPA DEVASTADA AL DESPEGUE ECONÓMICO DE LA POSGUERRA. LA PALANCA DE LA LUCHA CONTRA LA TUBERCULOSIS}

En julio de 1944 la Conferencia Monetaria y Financiera de Naciones Unidas se reúne en un complejo hotelero de Nueva Hampshire (EE.UU.), aunque asisten representantes de China y de la Unión Soviética, pronto se evidencia que la potencia que pretende imponer su hegemonía es Estados Unidos. Las soluciones adoptadas, conocidas como acuerdos de Bretton Woods, serán un reflejo directo de las políticas keynesianas, que marcarán la orientación económica de los inmediatos años de la posguerra en Europa, "regulación" que se definirá por una estrategia de crédito internacional dirigida por Estados Unidos sobre la Europa liberal a través del Plan Marshall (1947-1951), y cuyos principales beneficiarios serán Gran Bretaña, Francia, Dinamarca o Países Bajos, es decir, la Europa alineada con el eje Washington-Londres ya en los prolegómenos de la Guerra Fría. La consecuencia general de esta inyección de dinero será el inicio del despegue económico del viejo continente, y el desarrollo de una época de bonanza y crecimiento que no se interrumpirá hasta la crisis del petróleo, en 1973.

Como resultado de esta etapa de optimismo, consolidada en los años cincuenta, hemos de entender el objetivo de erradicar la tuberculosis, la poliomielitis o la viruela como una estrategia que, más allá de sus consecuencias prácticas económicas, sanitarias o sociales- despierta un ánimo simbólico de lucha por la vida en un contexto en el que todavía pesaban los traumas de la II Guerra Mundial, y en el que la Guerra Fría y la probabilidad de desastre nuclear 
significan una amenaza constante y real para la humanidad, por ello, marcadas por este nuevo espíritu, nuevas instituciones internacionales como la ONU, la OMS o UNICEF decidirán iniciar otro concepto de guerra, el de la lucha contra estas viejas pandemias.

La tuberculosis venía siendo uno de los principales azotes desde mediados del siglo XIX, su comportamiento había sido más silencioso que el de la viruela, pero no por ello menos importante en cuanto a constituir una seria amenaza para la salud del grueso de la población activa, y por tanto de la producción industrial. El joven especialista en tuberculosis, Halfdan Mahler (1923-2016), director de la OMS entre 1973 y 1988, ya lo advierte tras su experiencia en India durante los años cincuenta, un individuo podía estar años haciendo su vida sin dificultades a pesar de ser portador del bacilo, "such a nice unspectacular killer" (Brimnes 2011: 398). Es en este contexto de posguerra en el que situamos la acción de Estados Unidos, la Unión Soviética y la nueva Europa, y la decisión iniciar una cruzada contra las tres grandes enfermedades infecciosas que habían amenazado a la humanidad desde al menos el neolítico: la tuberculosis, la viruela y poliomielitis.

El plan internacional de erradicación de la viruela, surgirá de la primera propuesta y planificación del soviético Viktor Zhdanov (1914-1987) en 1958, durante la 11a Asamblea de la OMS. En cuanto a la poliomielitis, tras las ofensivas de vacunación de finales de los años cincuenta y principios de los sesenta, quedará muy mermada y circunscrita a las áreas más deprimidas del tercer mundo.

La lucha global contra la tuberculosis seguirá otros caminos. Hacía mediados de la década de los años cuarenta, la aceptación social de la vacuna contra la tuberculosis o BCG había sido muy diferente en Europa y América: en España, Alemania, Dinamarca, Suecia, Noruega, Unión Soviética, Uruguay, México o Brasil había sido bien recibida y aplicada desde antes de 1930, mientras que, ya a finales de los años veinte, Inglaterra y Estados Unidos se habían revelado como núcleo de resistencia contra la inmunización de Albert Calmette y Camille Guérin. Así, la BCG se implantará paulatinamente con éxito en el Norte de Europa desde 1927 y entre 1944 y 1947 se incorpora a un plan general de vacunación escolar vinculado al incipiente Estado de Bienestar, ya en plena postguerra europea. A partir de 1947, la Cruz Roja danesa, sueca y noruega diseñarán un gran programa de vacunación dirigido y liderado por Johannes Holm (1902-1990) -director del "Staten Serum Institute de Copenhage"- contra la tuberculosis, el objetivo es ponerlo en práctica en una de las áreas más 
devastadas por la guerra, el centro y sur-este de Europa, posteriormente, llevarán la misma campaña a India (Bryder 1999).

\section{LA FUERZA DEL INTERNACIONALISMO SANITARIO EN EUROPA Y LA LUCHA CONTRA LA TUBERCULOSIS: GÉNESIS DE LA INTERNATIONAL TUBERCULOSIS COMPAIGN (ITC)}

El panorama que los años de enfrentamiento bélico mundial dejan en el viejo continente es desolador. La tuberculosis se convierte en una enfermedad epidémica, cuya incidencia es dramática en el centro y el este de Europa. Las infraestructuras, y la población activa de Polonia, Yugoslavia o Hungría, sufren tales niveles de destrucción durante la guerra, que resulta imposible que produjeran una respuesta mínima al avance de la enfermedad. Ante esta situación de urgencia humanitaria, las recién creadas UNRRA (1943) y FAO (1945) protagonizan los primeros movimientos de cooperación internacional, centrados en Polonia, entre 1945 y 1946, (Black, 1986: 43-56) sobre todo, debido a la influencia de Sir John Boyd Orr (1880-1971), director de la FAO en aquellos momentos y, en especial, gracias al médico polaco Ludwik Rajchman (18811965), a la sazón, cofundador de UNICEF. Pero en concreto, será en el seno de la Cruz Roja danesa donde surja la primera iniciativa para organizar una gran campaña de vacunación que el New York Times calificará como la "cruzada médica más grande" de la historia (Brimnes 2007: 409).

Como decíamos, Johannes Holm ha experimentado con éxito la vacuna BCG en Dinamarca (Bryder 1999). Por ello, tras una primera aproximación en 1946, la Cruz Roja, y su director, el citado Holm, a su vez miembro de la OMS desde su fundación, se deciden a poner en marcha un proyecto mucho más ambicioso, una campaña de vacunación masiva en Polonia, Yugoslavia, Hungría, Rumania, Austria y Alemania. Como proclamará en 1948 el presidente de la Cruz Roja danesa, Commodore Kai Hammerich, en un intento de movilizar la solidaridad del pueblo danés durante un discurso radiofónico que recoge N. Brimnes (2007: 4008); si en otro tiempo los vikingos salieron con espadas, es el momento de que los vikingos salgan al mundo esta vez con agujas para combatir la tuberculosis, "Vikingefaer mod tuberkulosen", así rezó el lema del proyecto.

La campaña se inició con gran entusiasmo y apoyo popular, no obstante, según un documento de la OMS fechado en 1953 y firmado por Lydia B. Edwards (1905-2001), jefa de estudios de campo de la OMS, Carroll E. Palmer (19031972), director de la Oficina de Investigación sobre Tuberculosis (TRO) y Knut Magnus, asistente estadístico, cuando se emprendió el camino de la 
"International Tuberculosis Compaign" (ITC) se albergan todavía algunas "dudas científicas" a cerca de la BCG, sobre todo porque se duda que los métodos usados con éxito en Escandinavia ofrezcan los mismos resultados en otras latitudes y poblaciones, sobre todo por los problemas que puede presentar el transporte de la tuberculina y las reacciones alérgicas. En cualquier caso, la discusión será aclarada en el Congreso Internacional sobre BCG celebrado en París en 1948, cuya principal conclusión orienta la principal vigilancia hacia las condiciones del transporte y mantenimiento de la cadena de frío (Edwards, Palmer y Magnus 1953).

Reforzado el proyecto filantrópico tras la reunión de París, Dinamarca no será el único país en la empresa. A finales de 1947, Suecia y Noruega muestran gran interés en sumarse a la iniciativa. En noviembre de 1947, el conde Folke Bernadotte (1895-1948), presidente de la Cruz Roja sueca, visita Dinamarca, además, la "Norwegian Relief for Europe" y la "Europahjielpen" noruega se comprometen empujados por la presión que ya ejerce la crisis centroeuropea. En enero de 1948 se da el primer gran paso y se crea el Comité de Coordinación Escandinavo -Dinamarca, Suecia y Noruega-. (Holm, 1953) Pero para J. Holm no es suficiente, ya que pretende un mayor apoyo internacional, por ello, se reúne con Ludwik Rajchman y Robert Debré (1882-1978) (Black 1986: 39) con el objeto de impulsar la campaña con dos nuevos y poderosos socios. El resultado será óptimo, por una parte UNICEF firmará el primer acuerdo con el Comité Escandinavo el 12 de marzo, y por otra, lo hará la OMS, el 7 de abril de 1948 (Black 1986: 44). De este modo se pone en marcha definitivamente la "International Tuberculosis Compaign".

La OMS se compromete al asesoramiento científico, y al arbitraje de posibles problemas internos, y UNICEF aporta gran parte de la financiación, los bienes de equipo, medicamentos, suministros médicos y becas de formación especializadas para el personal sanitario. (Black 1986: 45) Holm será el coordinador de la ITC o "Joint enterprice". La cifra presupuestaria inicial asciende a 4 millones de dólares, el 50\% destinado a la campaña en Europa, y el otro 50\% a las campañas que se llevarán fuera del viejo continente. Copenhage se convierte el centro neurálgico de la ITC, desde donde se gestiona la contratación de personal, la dirección de aspectos educativos, publicitarios y estadísticos para Europa, Norte de África, Oriente Medio y Sureste Asiático. Por otra parte, se establece el un protocolo administrativo internacional para recibir asistencia de la ITC, previa solicitud y aprobación de la junta ejecutiva de UNICEF, que fijará dos tipos de programas de cooperación: a) campañas masivas dirigidas y sistematizadas por equipos médicos nacionales -la mayoría daneses, suecos o noruegos- en 
cooperación con el personal de la ITC; b) programas de demostración diseñados para instruir al personal nacional con el objeto de emprender campañas masivas en cada uno de sus países, programa que elegirán Italia, India y Pakistán.

Debido a las primeras fricciones entre las distintas organizaciones que impulsan la campaña, se creará el Comité mixto, con el objeto de resolver los posibles desacuerdos entre las diferentes instituciones cooperantes en la ITC. Las primeras desavenencias se darán en el seno de UNICEF, ya que algunos miembros no estarán de acuerdo con la idea de "empresa mixta". (Black 1986:41) Por influencia directa de Holm, la solución será la descentralización de las operaciones, es decir, la "Joint Enterprice" siempre colaborará directamente con las autoridades políticas y sanitarias de cada país, que, a su vez, nombrarán un director médico relacionado con la salud pública o la epidemiología, figura que ejercerá de puente entre los recursos, las necesidades de cada estado y la ITC. Por otra parte, el "Staten Serum Institute de Copenhage" asume la producción de tuberculina y de vacuna BCG para la mayoría de países en los que se desarrolla la ITC.

Por último, la idea de cooperación filantrópica, que pone de acuerdo bajo un objetivo común a todas estas instituciones citadas, será también el resultado de la influencia de Maurice Pate (1894-1965), primer director ejecutivo de UNICEF, aunque tampoco podemos olvidar figuras como la de Alfred Davidson (19112002), miembro destacado de la Internacional Refugee Organization (IRO), o el yugoslavo Berislav Borcic (1891-1977), que ejercerá de nexo imprescindible entre la OMS y UNICEF (Black 1986: 65).

Después de la primera experiencia en Polonia, los pasos de la recién creada ITC plantearán también el diagnóstico mediante rayos X, que se inicia en Makow, una pequeña población del sur de Polonia. A continuación, la campaña elige Sombor y Nobi Vrbas, ciudades medias del norte de Yugoslavia, actual Serbia. Pero los daneses encuentran grandes dificultades, sobre todo en cuanto a producción de tuberculina y vacuna, falta de electricidad y precariedad general de las infraestructuras (Holm 1951).

\section{DESARROLLO DE LA INTERNATIONAL TUBERCULOSIS COMPAIGN EN EUROPA, 1946-1951}

Tras estas experiencias iniciales en Polonia y Yugoslavia, y una vez firmados los acuerdos para la configuración internacional de los apoyos de la ITC, se establece un protocolo técnico de intervención, que consistía en formación de los equipos 
compuestos por enfermeras y médicos voluntarios, de entre 25 y 40 años de edad (Holm 1951), que recibirán un curso de formación específico y que por una parte, facilita el país que recibía la campaña y por otra, incorpora la misma OMS. Se fijan dos pruebas cutáneas iniciales: la del parche de Moro, indicada para los menores de 12 años, y la de tuberculina, para cualquier edad. A continuación, la ITC afrontará uno de los problemas decisivos, el de la cadena de frio: se crea un contendor específico -aunque también se usarán frascos termoeléctricos con hielo- y se implanta el transporte aéreo desde Copenhage, a temperaturas verificadas, siempre por debajo de los $4^{\circ}$, en plazos de entrega no superiores a 20 horas (Holm 1951).

Como dirá Johannes Holm, la campaña se organiza con "disciplina militar", en cuanto a planificación y preparación (Black 1986: 41). El principal objetivo es la vacunación de los menores de 18 años -aunque no exclusivamente- y supone al menos dos visitas a cada ciudad o pueblo, una primera para efectuar las pruebas de tuberculina, y una segunda, bien para administrar la BCG a la población sana, bien para comenzar los tratamientos sobre la población infectada. La dinámica de trabajo será muy intensa, se llegarán a efectuar 400 pruebas por hora, normalmente en escuelas, orfanatos, fábricas o centros relacionados con la salud. Además, la tuberculosis no será la única enfermedad de la que se ocupe la ITC, ya que también se actuará contra la sífilis, el tifus y la malaria (Black 1986: 56).

Durante los primeros meses de 1948, proliferarán las peticiones de ayuda a la ITC procedentes de diversos territorios europeos, como por ejemplo las de Hungría y Alemania, a través de las autoridades británicas, ya que en estos momentos controla gran parte de estas áreas, demandas a las que se sumarán, entre febrero y octubre del 1948, las de Finlandia, de nuevo Yugoslavia, Grecia, o Checoslovaquia. De modo que, en el verano de 1948, los equipos de médicos y de enfermeras procedentes de Dinamarca y Suecia ya se encuentran trabajando prácticamente en todo el centro de Europa, siendo Italia (septiembre de 1949) y Malta (febrero de 1950) los últimos países en incorporarse. Según el "Final report" de Holm, publicado por la OMS en octubre de 1951, en julio de 1950, la ITC se encuentra cooperando en 23 programas subvencionados, ha testado a 29.677.380 y vacunado contra la tuberculosis a un total de 13.874 .709 de personas, sin olvidar que ya ha traspasado las fronteras de Europa y comienza a iniciarse en India desde noviembre de 1948 o México, desde abril de 1950.

Aunque los programas cerrarán su actividad en Europa el 30 de junio de 1951, la ITC diseñará un plan de acción para el periodo posterior con el objeto de continuar con las pruebas de tuberculina y BCG, para ello se dejarán recursos 
importantes en cada país; vehículos, equipos y suministros médicos en determinados centros de vacunación de 18 países: Argelia, Austria, Ceilán, Checoslovaquia, Ecuador, Egipto, Finlandia, Grecia, India, Israel, Malta, México, Marruecos, Pakistán, Polonia, Siria, Túnez y Yugoslavia. También se prestará asistencia en la creación de laboratorios que produzcan BCG (Holm 1951).

Tras el final de la campaña en Europa, no existe acuerdo en las cifras de vacunaciones contra la tuberculosis: según N. Brimnes (2007), el programa inmunizará a 37 millones de personas, en cambio, para M. Black (1986), la cifra de inmunizaciones con BCG sería de unos 14 millones. Si tenemos en cuenta el "Final report of the International Tuberculosis Campign" de Holm, la cifra de vacunados alcanza los 6.696 .043 entre el 1 de julio de 1948 y el 30 de junio de 1951 (Holm 1951).

En definitiva, y lo más importante, es posible afirmar que la "Joint enterprice" desarrolla primera una gran campaña de salud pública general que "define una nueva era en salud pública internacional" (Holm 1953). A principios de la década de los cincuenta, la emergencia en Europa ha terminado, aunque cada país se comprometerá a continuar con labores de vigilancia, diagnóstico y vacunación durante varios años posteriores, tal es el caso de Checoslovaquia, Austria, Finlandia, Grecia, Polonia, Malta y Yugoslavia (Holm 1956).

\section{LA CREACIÓN DE LA TUBERCULOSIS RESEARCH OFFICE (TRO), 1948}

En pleno inicio de la campaña de vacunación, los dirigentes de la ITC y de la propia UNICEF observarán que es necesario abrir una vía de investigación científica que extrajera conclusiones de la acción práctica. La inmunización masiva ofrecerá una oportunidad única para investigar la tuberculosis. Por tanto, movidos por la iniciativa de Brock Chrisholm (1896-1971), director General de la OMS y del comité de tuberculosis de UNICEF, decidirán encargar la coordinación de dicha tarea al Director Médico del Servicio de Salud Pública de Estados Unidos, Carroll E. Palmer, que llegará a Europa en otoño de 1948. La toma de contacto de Palmer con la ITC consiste en la elaboración de una encuesta de la que se derivará a su vez un informe presentado al Comité Conjunto de Política de Salud de la OMS y UNICEF en octubre 1948. En este documento se reitera la oportunidad única ante la que se encuentra Europa para investigar la tuberculosis y la BCG. Para ello, Palmer propone la formación de un organismo científico permanente. En febrero de 1949, la propuesta se traduce en la creación de la "Tuberculosis Research Office" (TRO), con sede en Copenhage y apoyo financiero y logístico del gobierno danés. 
El primer campo de investigación en el que fijará su atención la TRO será la dificultad del enfoque internacional frente a las particularidades de cada país. La conclusión a la que se llega en septiembre de 1952, cuando todavía falta un año de trabajo, es la necesidad de una labor previa de investigación que incida en las condiciones específicas de cada país con respecto a la tuberculosis, dadas las enormes diferencias económicas y sociales que presenta cada nación, desequilibrios que Palmer considera vitales para un resultado óptimo de la campaña. Es muy importante tener en cuenta que para muchas de las zonas en las que ha trabajado la ITC es la primera vez que se realiza una estadística sobre salud pública o tuberculosis. La duda que se cierne es que la ITC desarrollará las pruebas de tuberculina y posterior vacunación según los criterios y resultados que se habían obtenido en Escandinavia, y los primeros resultados supondrán una primera decepción, ya que los saldos no serán los mismos en materia de respuesta alergénica e inmunización. Éste es el motivo que empujará a las autoridades médicas de la campaña a abrir una investigación más amplia que aborda las primeras dudas sobre alergias y cadena de frío. En el caso de Europa, los estudios sobre alergia a la tuberculina y/o a la BCG no empezarán hasta el otoño de 1950. La TRO iniciará 27 proyectos sobre unos 44.000 niños testados con tuberculina. Varios equipos de especialistas serán enviados a lugares tan diferentes como Grecia, Siria y Egipto, India y Ecuador con el objeto de comparar sus resultados con los obtenidos en Dinamarca y Finlandia. La conclusión será que tanto para los vacunados como para los no vacunados se presentará datos de morbilidad y mortalidad muy diferentes a los de Dinamarca y Finlandia.

El problema es evidente y la idea de fracaso menoscaba el optimismo inicial. La primera hipótesis que aporta Palmer y su equipo ante datos tan desalentadores apunta al test de tuberculina; "el material de alguno de los países de clima tropical indica que la reacción alérgica podía no ser debida a la alergia a la tuberculina y/o a la vacuna, sino la consecuencia de una deficiente conservación debido a las elevadas temperaturas o a la exposición a la luz" (Holm 1953). Las sospechas sobre de la cadena de frio surge de nuevo, sobre todo en determinadas áreas carentes de infraestructuras y recursos.

En el caso de India, el programa científico de la TRO cuenta con el apoyo del nuevo Estado indio. Las investigaciones se centrarán en la población rural, en Mandanapalle, Andhra Pradesh. Una de las dudas que en primer lugar despejará la TRO será que la vacuna se puede mantener durante 10 semanas a $2-4^{\circ} \mathrm{C}$ sin pérdida significativa de su potencia alergénica y que es posible almacenarla a una temperatura de $20^{\circ} \mathrm{C}$ durante un mes o a $37^{\circ} \mathrm{C}$, durante 5 días, causando, incluso 
en este caso, sólo una ligera reducción en el nivel de alergia a la tuberculina. Además, la BCG puede ser diluida 10 veces, o administrada por la mitad o el doble de la habitual, sin causar un cambio significativo en la reacción alérgica. Otro aspecto que se pone de manifiesto será la influencia de la profundidad con la que se inyecte la vacuna, aunque el nivel de alergia no se verá afectado, sí aumentará el tamaño de las lesiones locales o abscesos, reacción que puede confundir a los vacunadores.

En las investigaciones realizadas en Europa, uno de los aspectos más llamativos del estudio comparativo será la gran diferencia de reacciones alérgicas postvacunales que se dan en Dinamarca respecto de los países del sur. La primera conclusión se centrará en el distinto grado de exposición a la luz que sufre la vacuna en estos entornos, ya que se ha demostrado que la luz solar tiene un "efecto devastador" sobre la BCG, claramente observable en las reacciones postvacunales. En cualquier caso, el patrón alérgico que presenta la tuberculina seguirá siendo desconcertante, pues según los experimentos efectuados con una misma tuberculina en los países escandinavos, los resultados producen dos grupos, unos con fuerte reacción, otros con un nivel muy bajo. Si tenemos en cuenta el protocolo de la ITC, las personas que no tienen reacción se consideraban no infectadas y son vacunadas. Tras el estudio del equipo de Palmer, se abrirán dos nuevas dudas importantes: por una parte, ¿se está confundiendo la baja sensibilidad con la no infección?; y por otra, ¿hay que vacunar a aquellas personas que presentan bajo grado de sensibilidad? A finales de 1952 no habrá respuesta para estas preguntas, no obstante, los efectos generales de la BCG seguirán siendo óptimos desde el punto de vista de salud pública.

Pero a finales de los años cuarenta y principios de los cincuenta el contexto europeo presentaba un nuevo problema. Las consecuencias de la Guerra Fría se dejan notar en el viejo continente. De poco servirán los esfuerzos de UNICEF por mantener su neutralidad, ya que el macartismo comienza a investigar y a señalar a muchos cooperantes por el hecho de haber participado en las campañas de inmunización de Polonia o Hungría, lo cual acelerará el cierre de la ITC en los países que tras la Conferencia de Yalta han caído en la órbita soviética. Es significativo el texto de Gertrud Lutz-Frankhauser (1911-1995) tras su misión humanitaria en Polonia, que denuncia el deterioro del espíritu de la cooperación por efecto del nuevo enfrentamiento entre bloques y para la que "en un mundo enloquecido y devastado por la guerra, las relaciones amistosas deben ser mantenidas y la humanidad respetada. Las posibilidades en materia social, bienestar y salud son muy grandes. No debería de haber ninguna razón por la 
cual, las personas interesadas en la misma cosa y con un objetivo común no debieran trabajar juntos. En días como los nuestros no podemos abandonar el idealismo" (Black 1986: 45).

En este nuevo contexto, y tras el buen resultado obtenido en Europa, las expectativas de la Cruz Roja danesa, la OMS y UNICEF se orientan hacia Asia. China vive una situación epidémica como consecuencia de su Guerra Civil, que se prolonga prolongado hasta 1949 (Black 1986: 54) e India presenta cifras muy elevadas de infectados por el bacilo de Koch, por tanto, a pesar de la decepcionante falta de cooperación de algunos sectores políticos de Europa y sobre todo de Estados Unidos, la empresa continuará. La OMS seguirá siendo fiel a sus principios filantrópicos, y en su seno, la citada "Tuberculosis Research Office" (TRO), seguirá desarrollando su programa de investigación en Europa hasta 1953 y, simultáneamente en India, investigación que Carroll E. Palmer e I-Chi Yuan, director y director adjunto de la TRO, prolongarán en cooperación con el Servicio Nacional de Salud de Dinamarca, el "Staten Serum", la Asociación Nacional Antituberculosis de Dinamarca, la Asociación Nacional Antituberculosa de Finlandia, el Servicio de Control de Tuberculosis Gubernamental de Islandia y la "Union Mission Tuberculosis Sanatorium at Manapalle" de India. De hecho, en 1952, la TRO creará un laboratorio internacional con sede en Copenhage para estudiar la eficacia de la tuberculina y la BCG durante el desarrollo de la "Joint Interprice" (Yuan y Palmer 1953: 678). En definitiva, después de las presiones del macartismo y el consiguiente cierre de la ITC en Europa, las expectativas se centrarán en el desarrollo de la empresa común en una India que acaba de independizarse. Los objetivos generales del proyecto de investigación, promovidos por Palmer y Yuan, serán los siguientes: asistir a la ITC en sus años finales en cuanto a sus trabajos estadísticos y de formación del personal local; compilar las estadísticas sobre tuberculina y BCG, así como "manejar" dichas estadísticas. Hemos de decir también que hacia 1952, las investigaciones estadísticas de la TRO se centrarán en los países de Europa en los que la ITC ha intervenido, pero también en los que se ha proyectado intervenir fuera del ámbito europeo, es decir, los territorios del futuro Marruecos, Israel, los campamentos de refugiados palestinos, Siria, China, México y Ecuador, además, a partir de 1953, se incorporará la Colonia de Aden (sur de Yemen), Irán, Pakistán, Birmania, Formosa, Hong Kong, Filipinas, Malasia, Costa Rica, El Salvador, Jamaica y Trinidad.

Las conclusiones más importantes del estudio de la TRO constituirán la clave de las futuras campañas de vacunación con BCG en todo el mundo: será fundamental adaptar los programas de intervención a las diferentes condiciones 
sociales y climáticas de cada país, ya que lo que puede funcionar en Europa o Estados Unidos no será extrapolable a otras sociedades, sobre todo del tercer mundo (Yuan y Palmer 1953: 79). La campaña de India será la primera en beneficiarse de esta experiencia.

\section{LA CAMPAÑA DE INDIA (1948-63)}

Desde el éxito de las primeras vacunaciones en Polonia o los Balcanes, y al margen de la influencia de la Guerra Fría, la Cruz Roja danesa, ya desde antes de 1947 se plantea la posibilidad de continuar el proyecto fuera de Europa. La alianza de los países escandinavos en la lucha contra la tuberculosis, la estrecha cooperación con la OMS y el compromiso de UNICEF en materia financiera hacen que la campaña se exporte a la India de modo casi simultáneo al viejo continente.

La campaña de India será un "ejemplo temprano" (Brimnes 2007: 409) de los macroproyectos cooperación internacional que se lleven a cabo a partir de los años sesenta en el llamado tercer mundo, es decir, un gran ensayo de lo que va a ser la futura campaña de lucha contra la viruela, o como interpretan McMillen y Brimnes (2010: 180-209), un hito fundamental en el proceso de modernización de la medicina en el mundo entre los años cincuenta y sesenta del siglo XX.

Como advertirá Ludwick Raijman, primer presidente de UNICEF, la clave del éxito de la ITC, tanto en Europa como en India, será el ejemplo de vertebración y sinergia entre la OMS, UNICEF, la Cruz Roja danesa y sueca, y la "Europahjielpen" de Noruega. Vertebración y sinergia que Holm diseccionará en sus memorias, aclarando que el peso financiero de la campaña europea recaerá sobre las organizaciones escandinavas, mientras UNICEF se encargará de sufragar la diseñada en India (Brimnes 2007: 411).

En mayo de 1948, el joven gobierno de Nehru (1889-1964) reconoce que la tuberculosis ha alcanzado proporciones epidémicas, con unos 2,5 millones de casos activos y 1 millón de nuevas infecciones cada año. El hecho provoca una primera reunión en 1949 con la ITC aunque será la decisión de UNICEF la que impulse definitivamente la extensión del programa a India (Brimnes 2007: 410).

A partir de 1951, India estrechará su cooperación con UNICEF y la OMS, que aporta el suficiente asesoramiento técnico. Nace por tanto el concepto de contraparte, tan importante en el desarrollo posterior del sistema de cooperación internacional que en futuro van a protagonizar las Organizaciones No Gubernamentales de Europa y Estados Unidos. 
La campaña se organizará en dos fases: una primera (1948-55), cuyos objetivos, a su vez serán dos: por una parte, vacunar a unos 70 millones de niños, y por otra, alcanzar la inmunización de todos los menores de 25 años, es decir, unos 170 millones en total, cifras a las que se pretende llegar en 1955; y una segunda (1955-60), que incluso se prolonga hasta 1963, en la que se pretenden 200 millones de inmunizaciones.

El mecanismo que se emplea será el de brigadas de técnicos coordinados por personal médico aportado por la OMS y por el propio Estado indio. Es importante incidir en la figura de los "vacunadores laicos", que, a partir de 1950 desempeñan un papel decisivo, sobre todo porque se trata de indios formados para la campaña. (Brimnes 2007: 421) Indios que amortiguan el choque cultural y aceran la campaña a la población. A pesar de todo, Halfdan Mahler, que pasará diez años en India y dirigirá la campaña entre 1951 y 1955, advierte en su informe final que uno de los problemas que ha encontrado la campaña en su desarrollo, sobre todo en los últimos años, ha sido la apatía de los propios equipos profesionales (Brimnes 2007: 427). Obstáculo al que hay que añadir cinco grandes dificultades que hemos de tener en cuenta: las deficiencias en las comunicaciones; el rechazo cultural de la vacuna en gran parte de la población debido a la "ignorancia y la superstición"; la reacción anticolonial, de carácter nacionalista contra la campaña (MacMillan y Brimnes 2010: 190); el brote de propaganda anti-BCG localizado en Madrás, al que habrá que sumar la oposición a la vacuna de A. V. Raman y Chakravarti Rajagopalachari (1878-1972), quizá debido a la influencia británica, motivado a su vez por la preferencia de los antibióticos ante la BCG, y por último, la falta de personal cualificado, que se resolverá, aunque solo parcialmente, con la incorporación de los citados "vacunadores laicos" (Brimnes 2011: 401).

Sin duda, estas adversidades contribuirán a la dilatación de la campaña, que se alarga mucho más de lo previsto, hasta 1963, sobre todo debido a las referidas dificultades de comunicación que presentará la orografía y la enorme extensión del país, especialmente en el mundo rural, que explicarían la rectificación del primer objetivo, la pauta concreta de 30.000 vacunaciones al mes, que se tuvo que rebajar a 25.000 en los mejores casos ${ }^{1}$. No obstante, el conjunto de obstáculos no impedirá que en India, al final de la campaña, se haya logrado inmunizar a unos 200 millones de personas, la mayoría menores de 25 años. 


\section{PRIMERA FASE DE CAMPAÑA EN INDIA (1948-1955)}

La primera fase de la campaña se desarrolla en 26 estados de todo el país. La base demográfica de la que partirán los técnicos de la ITC será el volumen Primero del Censo de la India, de 1951, además de varios informes de los servicios de salud del gobierno. La vacuna contra la tuberculosis es el objeto prioritario de las autoridades sanitarias indias, a pesar de ello, hasta el momento de llegar la campaña, tanto la sociedad como muchos de sus profesionales médicos no son conscientes del problema social y económico que significa la tuberculosis y, como señalaba Mahler en su informe de 1955, viven "relajadamente" y en un ambiente de "laissare-faire". No existe disposición oficial que obligue a comunicar la tuberculosis, salvo en algunos estados. Los servicios sanitarios son muy precarios en los momentos en que los equipos de la ITC llegan a un país que, según sus propias autoridades, necesita unas 4.000 clínicas y 500.000 camas, pero en realidad no cuenta más que con 119 clínicas y unas 10.000 camas. Otros problemas con los que chocará la campaña serán la malnutrición endémica y la precaria higiene pública, que contribuirán en mucho a la proliferación de la enfermedad (Mahaler).

Desde febrero de 1949 a julio de 1951 se pone en marcha la campaña en tres estados piloto, en los que se inmuniza con BCG ya de forma masiva. Después de estos meses de trabajo, la ITC decide que las vacunaciones se centren en niños y jóvenes, pero también, y con especial atención, en varones de entre 25 y 40 años, que serán denominados "man-power" debido a su importancia en el desarrollo industrial como mano productiva. La formación de médicos, técnicos locales y de los equipos de vacunación, que se llevará a cabo entre febrero de 1949 y marzo de 1951, correrá a cargo del Estado indio y durante la primera fase la campaña se propone la integración de la vacuna en los programas generales de prevención de todo el país. Se crea una organización central de BCG, compuesta por un oficial médico superior, una oficina de publicidad, un asistente de suministros y especialista en estadística, además, se configuran los equipos de vacunación formados por un médico, 6 técnicos y personal auxiliar. Las vacunaciones serán efectuadas por enfermeras cualificadas. Según los planteamientos iniciales, se prevé que a finales de 1953 estén en funcionamiento 100 equipos de vacunación como mínimo y 10 más en 1954. El objetivo de estos equipos es efectuar 120.000 análisis de tuberculina al año (Mahler).

UNICEF se comprometerá a proporcionar equipos de vacunación, medios de transporte, un código de normas de trabajo y los suministros necesarios para el funcionamiento del laboratorio que producirán la vacuna en Guindy, en el 
estado de Tamil Nadu, al sureste del país. El Gobierno central ofrece el alojamiento del personal destacado por la ITC, así como el suministro de recursos locales necesarios, combustible, aceite, hielo o alcohol (Mahler).

Antes de la llegada de los equipos se diseñará una estrategia informativa que consistirá en reuniones con las personas influyentes que ejercen como elemento de confianza para la población, su función es explicar a la población las ventajas y el concepto básico de prevención e inmunización. A continuación, las poblaciones más grandes de cada área son visitadas por agentes informantes de la ITC que proyectarán películas, y tres días antes de la llegada de los equipos, se evalúa el estado de aceptación de la población.

Según Haldfan Mahler, en 1955 se efectúan 13 millones de pruebas y se vacuna a unos 4,5 millones de personas de 26 estados. La perspectiva para 1956-57 se fijará en unos 30 millones de vacunaciones anuales, con el horizonte de las125 millones de inmunizaciones, es decir, toda la población india de 1 a 25 años, a lo que hay que añadir 25 millones más de vacunaciones sobre la población denominada "man-power", como se observa, la mujer queda relegada a un segundo plano, en beneficio de la población activa masculina que trabaja directamente en la industria, la agricultura o los servicios, es decir, la campaña se revela como una estrategia económica, de gran contenido machista, tanto del nuevo gobierno indio como de las potencias occidentales implicadas (Holm 1953).

Durante esta primera fase, Mahler valorará que las autoridades locales centran en la vacuna la principal arma de control preventivo de la tuberculosis, aunque también "denuncia" la falta de interés y planificación del gobierno indio. Según el informe de Mahler, éste será a la postre el gran obstáculo para el óptimo desarrollo de la campaña. Por ello, en la Conferencia de BCG, de febrero de 1954, celebrada en Coimbatore, Tamil Nadhu, se pretende reestructurar la organización de los equipos con el fin de mejorar su eficiencia, para lo cual se incrementa en un $15 \%$ el presupuesto de la campaña y se decide que, aunque es preferible que los equipos estén liderados por médicos, se permite desempeñar esta función a hombres que hayan tenido una formación de 6 meses en materia de BCG y demuestren condición de liderazgo. Por otra parte, también se mejorará la formación de las técnicas, mujeres solteras, de entre 18 y 25 años, que han de superar 3 meses de formación. También se prestará especial interés en personal auxiliar, sobre todo el relacionado con el transporte, que hasta el momento ha sido uno de los aspectos más deficitarios. Tras la citada reunión de Coimbatore, serán 120 los equipos de BCG distribuido por todo el país, y para 
1957, se fijará el objetivo de 150 millones de pruebas que habrán de cubrir el total de la población india comprendida entre los 1 y 25 años de edad.

Durante 1954, el promedio de los 12 meses en toda la geografía india alcanzará 205.000 pruebas, lo cual supone un incremento del $70 \%$ con respecto a objetivo inicial. La cantidad de pruebas mensuales presentará importantes diferencias, ya que, mientras que las zonas menos pobladas y de dificultoso acceso no superan las 5.000, otras áreas llegarán a las 30.000 pruebas, con la excepción de las campañas de Delhi y Coimbatore, que registran hasta 90.000 tests de turbeculina mensuales. Según el "Informe final" de Halfdan Mahler, en la primera fase, la cobertura de BCG alcanzará un 75\% en el grupo de 1 a 6 años de edad, un $80 \%$ entre 7 y 15 , y de un 65\%, en el grupo comprendido entre 15 y 24 años. A su vez, el coste por test irá descendiendo hasta un $60 \%$ desde 1949 , que cada prueba costaba 0,72 rupias, hasta las 0, 12 en 1955 (Mahler).

Otra decisión importante a la que se referirá Mahler es que, tras los estudios de la TRO, el gobierno central fija el umbral de la reacción a la tuberculina en $6 / 8 \mathrm{~mm}$ desde el 1 de mayo de 1955 (Mahler).

El éxito de la campaña recaerá en el valor de las "personas clave" (Mahler), cuya función es popularizar el mensaje de la salud pública y de la vacunación en toda la población, tanto en el mundo rural como en el urbano y suburbano, también abrir el camino para afrontar otras enfermedades y otras campañas futuras, como la de la viruela, la poliomielitis o las de carácter venéreo.

Según Halfdan Mahler, hasta 1955, es decir en pleno ecuador de la "Joint Interprice", superada la primera fase de la ITC en India, se pueden extraer las siguientes conclusiones: 26 Estados de la India han desarrollado por primera vez una campaña de Salud Pública dirigida a la protección de "grupos de niños y adolescentes" contra la tuberculosis; entre 125 y 150 equipos de BCG dirigidos por 26 Oficiales, 120 líderes de equipo y 750 técnicos llegan a operar en 1955, con un promedio de 2,3 millones de pruebas y 0,8 millones de vacunas por mes; la media de cobertura epidemiológica en el grupo de edad comprendido entre 1 y 25 años será, aproximadamente del 65\%, lo cual, según Mahler, lejos de ser satisfactorio,

superará cualquier logro anterior en salud pública en India; el coste medio por operación será, un 200-300\% más barato que en otros proyectos comparables de salud pública, como ha sido hasta aquel momento el caso de la campaña contra la viruela; la capacitación de aproximadamente 800 técnicos y 150 médicos en la planificación general y la experiencia en la organización de un programa de salud de masas proporciona a cada Estado un núcleo vital de especialistas que en 
teoría garantizará la continuidad de la lucha contra la tuberculosis en India; otro logro importante de la ITC es que tuberculosis se empieza a considerar como un problema de salud pública en la mente de los médico, las autoridades públicas y el pueblo en general; y por último, la experiencia de la campaña masiva en un país con las enormes dificultades geográficas, económicas, sociales y culturales que presenta India en los primeros años cincuenta supondrá una apertura a la cooperación internacional futura (Mahler).

\section{SEGUNDA FASE DE LA CAMPAÑA EN INDIA (1956-1963)}

La segunda fase se inicia con la creación del Centro de Investigación de Tuberculosis (TRC) por iniciativa del Consejo Indio de Investigación Médica (ICMR), el gobierno del estado de Chennai, la OMS, el British Medical Research Council (BMRC) y el propio gobierno indio. En 1959, el Instituto Nacional de Tuberculosis (NTI) fijará su sede en Bangalore (Karnataka) con el objeto de desarrollar un proyecto de investigación (Amsith 2004:113-130) y un programa práctico de tuberculosis que pueda aplicarse en todas las partes del país mediante la capacitación de médicos y otros profesionales sanitarios.

En 1956 el indio P. Mohamed Alí sustituirá a Mahler en la dirección de la campaña, y se convierte en el responsable del desarrollo de la ITC hasta 1963. Esta segunda fase de la "Joint interprice" se caracterizará por una mayor involucración y compromiso del gobierno central con las organizaciones internacionales, es decir, las denuncias de Mahler no han sido en vano. No obstante, en abril de 1955 surgirá un nuevo problema, el movimiento anti-BCG rebrota a raíz de la publicación de un artículo que cuestiona la vacuna contra la tuberculosis en British Medical Journal. (MacMIllan y Bromnes 2010: 191) El hecho producirá consecuencias importantes, ya que durante el primer año de la segunda fase, P. Mohamed Alí tendrá que afrontar una caída drástica de las pruebas, que descienden en un 75\% según los objetivos previstos por la Campaña para 1956. El nuevo coordinador argumentará una serie de dificultades que explican el déficit: los efectos del monzón y una epidemia de viruela, aunque hará especial hincapié en la penetración social de la propaganda anti-BCG (Brimnes 2011: 397-407).

A finales de los años cincuenta, el panorama político seguía presentando serios obstáculos en India. La concepción social de la vacuna contra la tuberculosis adoptaba posiciones cada vez más beligerantes y sus detractores, influidos probablemente por las corrientes anti-BCG inglesas y estadounidenses son más fuertes. Sería interesante investigar qué intereses hay detrás del movimiento 
anti-vacuna, si se trata de una reacción oportunista de algunos sectores del nacionalismo indio y/o si se relaciona con el impacto económico que significa el auge y desarrollo de los antibióticos y su producción industrial.

En 1959, en Madras, Chakravarti Rajagopalachari funda "Swatantra", un partido conservador y tradicionalista poco a fin con la vacuna de Calmette y Guérin. La oposición a la controvertida inmunización contra la tuberculosis será cada vez más numerosa, también la vacuna contra la viruela había generado y genrará importantes críticas. Como advierten McMillen y Brimnes, Chacravarthi Rajagopalachari, líder conservador, utilizará el rechazo de la BCG como una estrategia para enfrentarse al gobierno central, es decir, la ITC será un símbolo perfecto para identificar y atacar la modernidad y el socialismo de Nerhu (MacMillan y Bromnes 2010: 180-209).

En 1960, P. Mohamed Alí manifestará su desánimo en unas declaraciones que reproduce Brimnes: "Casi no pasa un trimestre sin ningún incidente importante que altere incluso los planes más cuidadosamente trazados" (Brimnes 2011: 404). Aún así, hacia finales de 1962 y principios de 1963, se superan los 200 millones de pruebas que suponían unos 170 millones de inmunizaciones en toda India. En 1962, el gobierno de Nueva Delhi lanza su propio Programa Nacional de Control de Tuberculosis (National Tuberculosis Control Programme, NTCP), (Sandhu 2011: 143-150) que a partir de ese momento dirigirá las vacunaciones con BCG en en todo el país. Según Ch. Lahariya, una vez concluidos los programas de la ITC, y a pesar de las resistencia y de la campaña de desprestigio contra la vacuna, la vacuna será la única medida preventiva contra la tuberculosis disponible en India, además, se abrirá una vía de investigación en Chingelput, en el estado de Tamil Nadhu, que lleva a cabo un gran ensayo de BCG, el "Feasibility Study for TB Prevention Trial" o "Estudio de viabilidad para el ensayo de prevención de la tuberculosis". Este ensayo se inicia en 1968, consiste en el reclutamiento y seguimiento todos los casos, que se completará en 1987. El resultado de la investigación concluye que la vacuna BCG no ofrece una protección definitiva contra la tuberculosis pulmonar en adultos, a pesar de ello, se recomienda administrarla a una edad temprana, antes del final del primer año después del nacimiento (Lahariya 2014: 491-511).

Durante la década de los sesenta, quizá por influencia del movimiento anti-BCG, los programas de inmunización ya se encuentran en franco retroceso en beneficio de la quimioterapia -antibióticos-, sobre todo a partir de 1967, con el descubrimiento de la Rifampicina (Lahariya 2014: 505). 


\section{DESARROLLO DE LA CAMPAÑA DE SRI LANKA}

El avance de la campaña internacional contra la tuberculosis en Sri Lanka presentará importantes diferencias con respecto a India. La situación de la antigua Ceilán será mucho mejor desde el punto económico y de vista de salud pública. Los datos de tuberculosis son muy inferiores a los que presenta India a de finales de la década de los años cuarenta. Un año después de la independencia de la isla, en 1948, J.H.F. Jayasuriya funda la Asociación Nacional de Ceilán para la Prevención de la Tuberculosis. Su objetivo será la educación en salud y la recaudación de fondos, y su eje fundamental, la vacunación con BCG, que comienza en 1949 merced a la primera ayuda de la ITC, que se prolongará hasta 1956. Según el informe de Sir Bennet Hance, asesor médico del Departamento Británico de Relaciones de la Commenwealth, la campaña en Sri Lanka es todo un éxito, aunque el análisis de Jones Deeny, de la OMS, no coincide con esta visión tan positiva (Jones 2016: 514-533).

Después de 1956, los programas de vacunación contra la tuberculosis siguirán presentes en Sri Lanka, ya que, derivado de la intervención de la ITC, destaca el Proyecto Piloto de las Provincias del Noreste, impulsado en mayo de 1966.

En definitiva, según Margaret Jons, los programas de vacunación tendrán un "impacto positivo en la situación epidemiológica de la TBC" de Sri Lanka (Jones 2016: 529).

\section{CONCLUSIONES}

Como resultado de un contexto de expansión económica, consolidada entre los años cuarenta y cincuenta, hemos de entender que el objetivo de erradicar la tuberculosis, más allá de sus consecuencias prácticas, económicas, sanitarias o sociales, despertará un valor simbólico de lucha por la vida en un panorama en el que todavía pesan los traumas de la II Guerra Mundial, y en el que la Guerra Fría, y la probabilidad de desastre nuclear representan una amenaza constante y real para la humanidad, por ello, instituciones internacionales como la ONU, la OMS o UNICEF deciden iniciar otro concepto de guerra, el de la cooperación internacional destinada a la lucha contra las viejas pandemias, viruela, poliomielitis o tuberculosis.

La ITC se desarrolla entre 1947 y 1963 y constituye uno de los proyectos de inmunización más importantes de la historia del siglo XX. De desarrolla en 35 territorios o países: Argelia, Sri Lanka (Ceilán), Checoslovaquia, Ecuador, Egipto, Finlandia, Grecia, India, Israel, México, Marruecos, Pakistán, Polonia, 
Siria, Túnez, Yugoslavia, Malta, Polonia, Checoslovaquia, Hungría, Alemania, Austria, Italia, China, Colonia de Aden (sur de Yemen), Irán, Birmania, Formosa, Hong Kong, Filipinas, Malasia, Costa Rica, El Salvador, Jamaica y Trinidad.

Johannes Holm será el principal inspirador y coordinador de la ITC, tanto en Europa como otros continentes. El centro de operaciones administrativas, científicas y de gestión se establece en Copenhage y la producción de tuberculina y vacuna BCG es asumida por el "Staten Serum Institute" de Copenhage.

El objetivo de la ITC en Europa será la población menor de 18 años -aunque no exclusivamente- y supone al menos dos visitas a cada ciudad o pueblo, una primera para efectuar las pruebas de tuberculina, y una segunda, bien para administrar la BCG a la población sana, bien para comenzar los tratamientos sobre la población infectada.

El principal problema con el que chocará la ITC desde el punto de vista práctico y científico será la reacción alérgica a la prueba con tuberculina, ya que los presupuestos iniciales, desprendidos de las experiencias con BCG sobre la población danesa durante los años cuarenta no responden a los resultados de obtenidos en India. El otro gran problema con el que se encuentra la campaña mundial de BCG es la conservación de la tuberculina y la vacuna, que se resuelve con el establecimiento de un protocolo de transporte y conservación que supone la implantación de la cadena de frío de modo sistemático.

Según N. Brimnes, el programa de BCG en la postguerra europea concluye tras intervenir en 22 países de Europa y vacunar a 37 millones de personas, y 23 misiones sobre el terreno, en cambio, para $\mathrm{M}$. Black, la cifra de inmunizaciones con BCG es de unos 14 millones, y el Final report of the International Tuberculosis Campign, de Johannes Holm, fija la cifra de vacunados en 6.696.043 entre el 1 de julio de 1948 y el 30 de junio de 1951.

Dado que por primera vez se produce una campaña masiva con BCG en el mundo, según la OMS será necesario aprovechar la oportunidad como gran campo de investigación científica sobre tuberculosis y BCG, por este motivo se creará en 1948 la "Tuberculosis Research Office" (TRO) bajo la dirección de Carrol E. Palmer.

La ITC se desarrolla en India entre 1948 y 1963, a lo largo de dos fases: la primera, dirigida por Halfdan Mahler, de 1948 a 1955; y la segunda, de 1956 a 1963, dirigida por el indio P. Mohamed Alí, periodo en el que destaca el movimiento anti-BCG de Chacravarti Rajagopalachari en Madrás. 
El resultado final de la campaña en India será positivo, ya que logrará la vacunación de unos 170 millones de personas de entre 0 y 40 años de edad. También se consigue el establecimiento de la BCG como recurso preventivo del gobierno central.

La campaña de vacunación desarrollada en India tendrá un marcado sesgo sexista al centrar parte de su actividad sobre los "man-power", varones de entre 25 y 40 años de edad que constituían el gran contingente de la población activa y de cuya salud dependía el devenir económico del país.

La ITC y la OMS fijarán una estrategia de intervención centrada en información, propaganda para la inmunización y organización de equipos de vacunación formados por médicos, enfermeros y personal técnico, que sirvirá de modelo en otras campañas por todo el mundo.

A pesar de las dificultades, las campañas internacionales de BCG en Europa e India, desarrollada entre 1947 y 1963, tendrán un resultado satisfactorio general. Su importancia radicará en dos grandes aspectos trascendentales en el siglo XX: por una parte, supone un gran ensayo para lo que inmediatamente será la gran campaña global de erradicación definitiva de la viruela y polio de la OMS en los años sesenta; por otra, inaugura la fase de "cooperación internacional y desarrollo" que tanta importante tendrá en cuanto a las relaciones de Europa y Estados Unidos respecto del tercer mundo, no sólo gracias a la Cruz Roja internacional, la OMS o UNICEF, sino también a un nuevo concepto, el de las Organizaciones No Gubernamentales (ONG) en todo el planeta.

\section{BIBLIOGRAFÍA Y DOCUMENTACIÓN}

AMRITH, Sunil. (2004): “In search of a 'Magic Bullet' for Tuberculosis: South India and Beyond", Social History of Medicine, 17(1), pp. 113-130. Consultado: 28 de enero de 2018 (https://academic.oup.com/shm/article/17/1/113/1628706/In-Search-of-aMagic-Bullet-for-Tuberculosis-South).

BLACK, Maggie. (1986): The children and the Nations. The story of Unicef. Sidney, UNICEF, pp. 37-62. Consultado el 2 de diciembre de 2017 (https://www.unicef.org/about/history/files/Child-Nation-M-Black-Ch02p37-62-milk-fat-bread.pdf). 
BRIMNES, Niels. (2011): "Another vaccine, another story: BCG vaccination against tuberculosis in India, 1948 to 1960", Ciencia \&Saúde Coletiva, 16, pp. 397-407.

BRYDER, Linda. (1999): "We shall not find salvation in involution: BCG vaccination in Scandinavia, Britain and the USA, 1921-1960", Social Cience \& Medicine, 49, pp. 1157-1167.

BRIMNES, Niels. (2007): "Viking against tuberculosis: the international tuberculosis compaign in India, 1948-1951”, Bull. His. Med., 81, pp. 407-430.

EDWARDS, Lydia B., PALMER, Carroll E. y MAGNUS, Knut. (1953) "BCG vaccination". Studies by the WHO Tuberculosis Research Office, Copenhage. Geneve.

HOLM, J. (1951): "Final Report of the International tuberculosis Campaign, July 1, 1948 to, 1951 Inception, Organizarion, PR, Funding, Supply, personnel, statictics, country operations results. Part 1." Octuber. Consultado: 18 de diciembre de 2017 (http://www.cf-hst.net/unicef-temp/DocRepository/doc/doc449141.PDF).

JONES, Margaret. (2016): "Policy Innovation and Policy Pathways: Tuberculosis Control in Sri Lanka, 1948-1990”, Med. Hist., 60(4), pp. 514-533.

LAHARIYA, Chandrakant (2014): "A brief history of vaccines \& vaccination in India”, Indian J. Med Res., 139(4), pp. 491-511. Consultado: 21 de diciembre de 2017 (https:/www.ncbi.nlm.nih.gov/pmc/articles/PMC4078488/).

MACMILLAN, Christian W. y BRIMNES, Niels (2010): "Medical Modernization and Medical Nationalism: Ressistence to Mass Tuberculosis Vaccination in Postcolonial India, 1948-55. Comparative Studies", Society and History, 52, pp. 180-209.

MAHLER, H. Final report on India BCG by H. Mahler", WHO/UNICEF campaign. Consultado: 14 de enero 2018 (http://www.cf-hst.net/UNICEFTEMP/Doc-Repository/doc/doc450078.PDF).

"Report of Tuberculosis Research Office, Copenhagen", WHO. Consultado: 16 de diciembre de 2017.

(http://apps.who.int/iris/bitstream/10665/128561/1/EB11_12_eng.pdf). 
SANDHU, Gursimrat K. (2011): "Tuberculosis: Current Situation, Challenges and Overview of its Control Programs in India", J. Glob. Infect. Dis., AprJun, 3(2), pp. 143-150. Consultado: 21 de enero de 2018 (https://www.ncbi.nlm.nih.gov/pmc/articles/PMC3125027/).

YUAN, I-Chin y PALMER, Carroll E. (1953): "The WHO Tuberculosis Research Oficce -A Reviw of the First Four Years-" Public Health Reports, 68; 7, 7 july, 678-79.

Recibido: 21 de octubre de 2019

Aceptado: 15 de noviembre de 2019

Mariano Monge Juárez es doctor en Historia contemporánea por la UA, licenciado en Geografía e Historia por la Universidad de Alicante y licenciado en Antropología Social por la Universidad Miguel Hernández. Actualmente es profesor de Historia contemporánea de la Universidad de Murcia y ha sido investigador en la Univesidad de Alicante. Es autor de una biografía de Sabino Arana, así como de varios artículos y libros sobre contemporaneidad, biopolítica e historia epidémica. mongejuarez@um.es 